\title{
Semantic processing during sleep
}

\author{
BENNY SHANON \\ Hebrew University of Jerusalem, Jerusalem, Israel
}

\begin{abstract}
Sleeping subjects were presented with simple nouns and they were asked to respond to them. Microswitch pressings and distribution of $\mathrm{K}$ complexes indicated that subjects could report to nonemotive verbal information during sleep. The underlying processes were examined on the basis of generalizations to distractors related to the subjects in sound or meaning (within or across languages).
\end{abstract}

Casual observations suggest that people can react selectively to meaningful stimuli during sleep. People wake to the sound of their names, but not to those of others, and mothers wake to the cries of their own babies, but not to those of other babies. These observations raise two questions. The first question has to do with the range of the phenomenon at hand: Is it limited to an extremely small set of items characterized by high emotive value, or is it encountered with other types of information as well? The second question focuses on the underlying cognitive mechanisms: Are they similar to the semantic processes that operate in wakefulness, only with a reduced level of activation (i.e., when asleep, one is covered with a mental blanket, so to speak, which makes the access of information more difficult but does not alter the manner in which it is processed once detected)? Or, perhaps, they are qualitatively different and considerably more limited (i.e., involve the activation of a limited number of preset "cells" each triggered by very specific signals only). The answers to the two questions are related: If the phenomena under consideration are limited to emotive inputs, a simple detection mechanism may be sufficient; if, however, the range of the phenomenon is more extended, genuine semantic processing may be needed. Note, however, that the two answers are independent: Sleeping subjects might respond to nonemotive items, yet the semantic machinery involved can be rather limited.

Oswald, Taylor, and Treisman (1960) presented sleeping subjects with lists of proper names all during their sleeping night. The subjects were asked to respond to preassigned items on the list, other than their own names, by pressing a microswitch. The distribution of these overt responses, as well as the distribution of

This experiment was conducted at the Sleep and Dream Laboratory at the Boston State Hospital. It could never have been executed without the kind help of Ernst Hartmann, who has allowed me to use the facilities of his laboratory. I am also grateful to him for helpful discussions and encouragement. Thanks are extended to Jack Carroll, James Cravens, Thomas Haggestad, Ken Parulsky, Vincent Stanton, and Greta Unger for their help in the preparation and execution of the experiment, and to Maya Bar-Hillel and David Navon for comments on the manuscript. awakening to the sound of the stimulus and the occurrence of $\mathrm{K}$ complexes (which are taken as indicative of shifts in attention), suggested particular attentiveness to the assigned names. General attentiveness to proper names was also found by McDonald, Schicht, Fratier, Shallenberger, and Edwards (1975), who did not ask subjects for any overt response, but measured plethysmographic changes in the finger, variations in heart rate, and occurrence of $\mathrm{K}$ complexes. That sleeping subjects are indeed capable of making discriminations and indicating their responses by the pressing of a microswitch was subsequently reaffirmed with nonverbal material (cf. Williams, Murlock, \& Murlock, 1966).

Other techniques used in the study of sleeping subjects reactions to verbal stimuli included hypnotic suggestion and manipulation of dream content. Evans, Gufstafson, O'Connell, Orne, and Shor (1970) showed that sleeping subjects generated motor responses in reaction to cued words that were introduced by hypnotic suggestion during the ascending phase of Stage 1 of sleep. Berger (1963) presented spoken proper names to subjects while they were asleep. Postexperimental matching of the names with the associations of the dreams indicated that the subjects incorporated the stimuli into their dreams.

The present study follows the Oswald et al. (1960) technique while considering full-fledged semantic stimuli (which proper names are not). Its underlying rationale was the following. First, by way of answering the first question introduced above (regarding the range of the phenomenon at hand), it is sufficient to find nonrandom responses to common nouns lacking particular emotive content in order to conclude that the sleeping mind can react to nonspecific verbal information. Second, by way of answering the second question (regarding processing), various types of distractors were introduced. Generalization to the distractors is expected only if some processing is involved; further, the kind of generalization made would be indicative of the nature of this processing.

\section{METHOD}

Subjects

Five M.I.T. undergraduates participated in the study. All 
were native speakers of English with a college-level knowledge of French; they were paid $\$ 10 /$ night.

\section{Materials and Procedure}

Tapes consisting of words appearing at the rate of one per $5 \mathrm{sec}$ were played to subjects all during the night. The test tape contained 10 lists of 48 words (tokens) each. The words belonged to the following categories: English words that served as a base (for example, rabbit), synonyms of these words (bunny), English words sounding like the base words (habit), and French translations of them (lapin). The base words were all mono- or bisyllabic common concrete nouns (mean frequency of 118 in the Kucera \& Francis, 1967, count). No words were homophones in either English or French; the same constraints were met for synonyms and sound-alikes, as well. The soundalikes were generated by substituting one consonant of the base word with another. The French words were all common monoor bisyllabic concrete nouns with no homophones; none of them was a cognate of the English words or a homophone thereof (and vice versa). Thirty-two of the items on each list consisted of eight quadruples constructed in this manner. The other 16 items were French words; these served as fillers so as to equate the probability of occurrence of words in the two languages. The same words appeared on all lists, with each word appearing only once on each list. The lists differed only in the order of words, which was always random.

The words were read by a female, and the test tape was played at a medium-high volume, about that of a loud conversation. When the 40-min tape was terminated, it was rewound and played again and again until the end of the experimental session. The test tape was preceded at the very beginning of the session by a warm-up consisting of the base words only. This tape was 80 words long (a random ordering of 10 occurrences of the 8 base words) and was played three times in a row.

Each subject slept in a comfortably arranged individual room. Silver-coated surface electrodes were planted on the subject's head and connected to a Grass electroencephalograph (Model 8) set at a recording speed of $10 \mathrm{~mm} / \mathrm{sec}$. Seven recording channels were used: Two channels recorded the subject's EEG activity (right parietal and right occipital), one recorded muscle activity (chin to chin), and two recorded eye movements. A left parietal electrode served as a ground. The sixth channel was connected to a microphone located by the subject's bed. The microphone was triggered by the onset of the experimental words and activated the pen that marked a temporal reference point. The last channel was connected to a microswitch fastened to the subject's right hand all during the night. The microswitch was designed so that its activation would not require too much pressure but would not be too easy, either. It was taped between the subject's thumb and hand and could not be activated by random gross body or hand movements.

The recorded probes were transmitted to the subject via an intercom system. The experimenter listened to the tape directly and watched the recording; all during the night. He also wrote down the actual words on the recording paper.

At the beginning of the experimental session, the subjects were told that they were about to participate in a study of verbal processing during sleep. After the electrodes and the switch were placed on their heads and hands, respectively, the subjects were presented a pair of English words, chosen at random by the experimenter. They were asked to press the microswitch whenever they heard these words and were given the trial lists. The experiment proper started between midnight and 1:00 a.m. and ended with the subject's free awakening between $6: 00$ and $7: 00$ a.m.

\section{RESULTS}

All subjects fell asleep, with the mean time elapsing between the beginning of the experimental trials and the onset of sleep being $45 \mathrm{~min}$. The mean net length of the experimental session during sleep (including intersession sporadic awakenings) was $4 \mathrm{~h} 50 \mathrm{~min}$. All subjects had a REM period. The patterns of sleep, however, were not regular, and at times, not usual. Stage 2 of sleep was prolonged, and the REM periods were few, short, and delayed until the latter part of the night.

The data are based on 10 subject-nights. They were analyzed by two independent judges: One determined whether the subject was asleep at the time of the responses at hand, and the other classified these responses according to the different word categories. Responses were counted only when it was established that the subject was physiologically asleep when they occurred, as well as $30 \mathrm{sec}$ before and after. Two independent measures were subject to statistical analysis: rate of extent of microswitch pressing, and distribution of the $\mathrm{K}$ complexes. Due to a technical failure, the first analysis is based on nine subject-nights only.

A total of 36 pressings by sleeping subjects were observed. Only in 1 of the 10 subject-nights comprising the study were no microswitch pressings collected. The number of pressings in the other nights varied from one to eight. Twenty-five pressings were in response to the target words, two to the sound-alikes, one to the synonyms, three to the French translations, and five to the random English words. ${ }^{1}$ These numbers reflect an overall very low response rate: They constitute approximately $1.78 \%, .14 \%, .07 \%, .21 \%$, and $.35 \%$, respectively, of all possible responses. The rate of response to the target words was significantly higher than that to any of the other four word categories $[\mathrm{t}(8)=3.70, \mathrm{p}<.005 ; \mathrm{t}(8)=4.43, \mathrm{p}<.005 ; \mathrm{t}(8)=4.40$, $\mathrm{p}<.005$; and $\mathrm{t}(8)=3.25, \mathrm{p}<.01$, for the comparison with the sound-alikes, synonyms, translations, and random words, respectively]. The differences among the rates of response associated with the other categories were not significant.

The distribution of the pressings in the different stages of the subjects' sleep was also noted. Most responses were collected during Stage 2 of sleep (19 responses) or in the shift between it and Stage 1 (3 responses). Seven responses were collected during D sleep, five in Stage 3, and two in Stage 1. This relative distribution was kept within the responses to the target words; the responses to the other word categories were too few to show any significant pattern.

The distribution of the $\mathrm{K}$ complexes was 71 to the target words, 55 to the sound-alikes, 40 to the synonyms, 24 to the French translations, and 54 to the English random words. (The respective percentages are: $4.09 \%, 3.79 \%, 2.76 \%, 1.66 \%$, and $3.72 \%$.) ${ }^{2}$ The rate of response to the target words was significantly higher than that to the synonyms and the French translations $[t(9)=3.08, p<.05$, and $t(9)=3.32, p<.01]$; the 
respective comparisons with the sound-alikes and the random words were marginally significant $[\mathrm{t}(9)=1.44$, $\mathrm{p}<.10$, and $\mathrm{t}(9)=1.49, \mathrm{p}<.10]$. The rate of response to the French words was significantly lower than that to the random words $[t(9)=2.55, \mathrm{p}<.05]$; the respective comparison with the synonyms was marginally significant $[\mathrm{t}(1,9)=2.18, \mathrm{p}<.10]$. No other values were significantly different from each other.

In three cases subjects awoke or moved drastically upon the reception of the target word. A similar response was also observed once in conjunction with a French translation.

The data were too scant to warrant detailed intersubject analysis. An examination of the raw data for each word category and for each dependent variable showed no effects of either habituation or learning for the same subject across different nights.

\section{DISCUSSION}

The small number of testings, coupled with the overall low frequency of the responses, weaken whatever conclusion is to be drawn on the basis of the present data. The obvious limitations of the present study notwithstanding, it does suggest some interesting observations. First, it gives a positive answer to our first question: Subjects emitted more responses to the targets, even though those responses were devoid of particular emotive significance. This result is interesting in light of previous suggestions regarding a dependency of discriminations during sleep on the emotive value of the stimulus: intrinsic (i.e., particular to the meaning of the stimuius or its familiarity) or extrinsic (i.e., introduced via a manipulation of the subject's motivation or by punishment) (cf. Granada \& Hammack, 1961; Weinberg, 1966; Williams et al., 1966; Zung \& Wilson, 1961).

The answer to be given to the second question is much more tentative. The lack of generalization on phonological and semantic grounds suggests that the processes involved are of a limited nature. Specifically, on both measures examined, performance on both homophones and synonyms was no different from that associated with the baseline. Nonspecific processing of the words would have generated both phonological and semantic confusions. These observations suggest that sleeping subjects respond to words by setting prepared "cells" that are sensitive to limited and specific information. (The nature of this information, as already noted, is, however, not constrained.)

The distribution of the $\mathrm{K}$ complexes exhibits one pattern that suggests that processing was nonetheless not that simple. The number of $\mathrm{K}$ complexes associated with the French words was significantly smaller than that associated with the random English words. This indicates that sleeping subjects are discriminative of high-order characteristics of verbal material, such as the language in which it appears. The subjects were instructed to respond to specific English words; as a consequence, they were more attentive to words in that language and tended to ignore words in French. The ability to discern between the language membership of words is attributed to an early peripheral mechanism, preceding the full identification of the word in question. $^{3}$

\section{REFERENCES}

BERGER, R. J. Experimental modification of dream content by meaningful verbal stimuli. British Journal of Psychiatry, 1963, 109, $722-740$.

Evans, F. J., Gustafson, L. A., O’Connell, D. N., Orne, P. T., \& Shor, R. E. Verbally induced behavioral responses during sleep. Journal of Nervous and Mental Disease, 1970, 150, 171-187.

Granda, A. M., \& Hамmack, J. T. Operant behavior during sleep. Science, 1961, 133, 1485-1486.

Kucera, H., \& Francis, W. W. Computational analysis of present-day American English. Providence, R. I: Brown University Press, 1967.

McDonald, D. G., Schicht, W. W., Fratier, R. B., Shallenberger, H. D., \& EDwards, D. J. Studies of information processing in sleep. Psychophysiology, 1975, 12, 624-629.

Oswald, I., Taylor, A. M., \& Treisman, A. M. Discriminative responses to stimulation during human sleep. Brain, 1960, 83, 440-453.

Treisman, A. M. Verbal cues, language, and meaning in selective attention. American Journal of Psychology, 1964, 77, 206-219.

WEINBERG, H. Evidence suggesting the acquisition of a simple discrimination during sleep. Canadian Journal of Psychology, 1966, 20, 1-11.

Williams, H. C., Murlock, H. C., \& Murlock, J. V. Instrumental behavior during sleep. Psychophysiology, 1966, 2, 208-216.

Zung, W. W. K., \& Wilson, W. P. Responses to auditory stimulation during sleep: Discrimination and arousal as indicated with electroencephalography. Archives of General Psychiatry, $1961,4,548-562$.

\section{NOTES}

1. In order to keep the number of items under comparison equal, values associated with random words were based on a subset constructed as follows. Words that appeared five items before the target words were checked; if they belonged to one of the experimental word categories, one still earlier word was checked. This procedure was continued until a random set equal in size to that of all other word categories was assembled.

2 . The absolute rates of occurrence of the $\mathrm{K}$ complex should be viewed with a qualification in mind. In Stages 3 and 4 of sleep, the complex is not very distinct; the actual rates of occurrence, then, might have been higher than the ones reported here. It is assumed that this qualification applies equally to the different word types, and that the discussion of relative distributions is not affected by it.

3. In this conjunction, note Treisman's (1964) findings that subjects attending to one of two different messages can still detect some features of the message in the nonattended channel: Shift in language is one of these features.

(Received for publication August 1, 1979.) 